\title{
Ameliorative effects of melatonin and zinc oxide nanoparticles treatment against adverse effects of busulfan induced infertility in male albino mice
}

\author{
Amoura M. ABOU-EL-NAGA ${ }^{1}$; Shaker A. MOUSA ${ }^{2}$; FAYeZ ALTHOBAITI ${ }^{3}$; EMAN FAYAD ${ }^{3, *}$; ENGY S. FAHIM ${ }^{1}$ \\ 1 Department of Zoology, Faculty of Science, Mansoura University, Mansoura, Egypt \\ 2 The Pharmaceutical Research Institute, Albany College of Pharmacy and Health Sciences, Rensselaer, USA \\ 3 Department of Biotechnology, Faculty of Sciences, Taif University, Taif, Saudi Arabia
}

Key words: Busulfan, Melatonin, Zinc oxide nanoparticles, Ultrastructure, Testis, Mice

\begin{abstract}
Testicular damage is one of the most hazardous effects as it's associated with azoospermia. Busulfan (Bu) is a highly toxic chemotherapeutic drug that affects testis. Thirty male Swiss albino mice divided into six groups of 5 animals each. Control (oral 0.9\% saline daily for 75 days); Mel (20 mg/kg/day orally for 30 days); ZnO NPs ( 5 mg/kg/day i.p. for 30 days); BU (single i.p. injection of $40 \mathrm{mg} / \mathrm{kg}$ and then left for 45 days); BU + Mel (single $40 \mathrm{mg} / \mathrm{kg}$ dose of BU and left for 45 days followed by $20 \mathrm{mg} / \mathrm{kg} /$ day Mel for 30 days); BU + ZnO NPs (single dose of $40 \mathrm{mg} / \mathrm{kg}$ of BU and left for 45 days, then $5 \mathrm{mg} / \mathrm{kg}$ /day ZnO NPs for 30 days). Preparation and Characterization of ZnO NPs. Specimens from testis prepared for ultrastructural investigations using TEM after Masson's trichrome and toluidine blue staining. BU induced histological and ultrastructural damage of the testis. Moreover, the present results could be concluded that Mel or ZnO NPs can protect the testicular tissue against ultrastructural alterations induced by BU by its antioxidant and anti-apoptotic effects.
\end{abstract}

\section{Introduction}

Infertility is a serious health problem around the world (Inhorn and Patrizio, 2015). Infertility can be caused by many medications such as chemotherapies and fertility is often impaired after chemotherapy (Delessard et al., 2020; Poganitsch-Korhonen et al., 2017). Deleterious in germ cells was detected due to the exposure to alkylating agents, such as cyclophosphamide, procarbazine, and busulfan (Delessard et al., 2020).

Busulfan (BU) is an alkylating agent that induces deoxyribonucleic acid (DNA) damage impairing DNA replication ( $\mathrm{Li}$ et al., 2018). Therefore, BU is a highly toxic chemotherapeutic agent that results in adverse effects on gonads (Mirhoseini et al., 2014). After one or two intraperitoneal injections, BU can effectively consume most spermatogonia. Thus, it is important to use antioxidants to treat infertility related to BU (Brinster et al., 2003).

Melatonin (Mel.) is a highly conserved molecule synthesized in all organisms. It is the chief secretory product of the pineal gland and was recently reported to be a

*Address correspondence to: Eman Fayad, e.esmail@tu.edu.sa Received: 02 June 2021; Accepted: 23 July 2021 powerful antioxidant (Gurunathan et al., 2020; HaghiAminjan et al., 2018). In both in vitro and in vivo studies, Mel protected tissues against oxidative damage caused by various free radicals. One of the protective mechanisms that might be related to Mel is anti-apoptotic effects (HaghiAminjan et al., 2018).

Melatonin (N-acetyl-5-methoxytryptamine), the chief secretory product of the pineal gland in the brain, is well known for its functional versatility. In hundreds of investigations, melatonin has been documented as a direct free radical scavenger and an indirect antioxidant, as well as an important immunomodulatory agent. The radical scavenging ability of melatonin is believed to work via electron donation to detoxify a variety of reactive oxygen and nitrogen species, including the highly toxic hydroxyl radical. It has long been recognized that the damaging effects of ionizing radiation are brought about by both direct and indirect mechanisms. The direct action produces disruption of sensitive molecules in the cells, whereas the indirect effects $(70 \%)$ result from its interaction with water molecules, which results in the production of highly reactive free radicals such as $\cdot \mathrm{OH}, \cdot \mathrm{H}$, and eaq- and their subsequent action on subcellular structures. The hydroxyl radical scavenging ability of melatonin was used as a rationale to determine its radioprotective efficiency (Reiter et al., 2004). 
Melatonin is a hormone produced naturally by the testes, retina, ovary, skin, and intestine (Chen et al., 2016). Melatonin is involved in the regulation of various biological pathways, including hormone secretion and modulation, reproduction, and circadian rhythms, according to previous research (Kim et al., 2012; Wang et al., 2012; Maroufi et al., 2020). Furthermore, melatonin has been demonstrated to have an effective anticancer effect on a variety of cancer cells with no adverse effects (Wang et al., 2012; Maroufi et al., 2020). Melatonin's actions are mediated by receptor-dependent or receptorindependent mechanisms (Maroufi et al., 2020; Witt-Enderby et al., 2006). Melatonin receptors MT1 and MT2 suppress many signaling pathways involved in cancer growth, including phosphoinositide 3-kinases (PI3K) and mitogen activated protein kinase (MAPK) pathways, as well as induce apoptotic pathways in cancer cells (Kong et al., 2008; Mao et al., 2010). Furthermore, when taken alone or in conjunction with therapeutic drugs, melatonin has been shown to have anticancer effect in hormone-dependent cancers (Menéndez-Menéndez and Martínez-Campa, 2018; Naja et al., 2019). Toxicity, cellular DNA breakage, and lipid peroxidation are all reduced by melatonin. Melatonin's ability to scavenge free radicals implies that it could be employed as a dependable protective medication in radiotherapy (Tan et al., 2007).

On the other hand, zinc ( $\mathrm{Zn}$ ) exists at high concentrations in testes of vertebrates comparable to the liver and kidney (Fallah et al., 2018). In addition, Zn could decrease testicular damage caused by many chemotherapeutic agents such as cyclophosphamide (Torabi et al., 2017). During early spermatogenesis, $\mathrm{Zn}$ is collected in the testes, and this plays a significant role in the meiosis division of germinal cells (Elgazar et al., 2005). Additionally, Zinc Oxide Nanoparticles ( $\mathrm{ZnO} \mathrm{NPs}$ ) is a new type of material with outstanding biological properties and no toxicity (Suresh et al., 2018). It seems to have great potential for effective cell and molecular selection through certain obstacles in many diseases (Keerthana and Kumar, 2020). The important characteristics of $\mathrm{ZnO}-\mathrm{NPs}$ are antioxidant, antimicrobial, anticancer, antiinflammatory activities (Madhumitha et al., 2016; Umar et al., 2019). Therefore, the present study evaluated the protective role of Mel or $\mathrm{ZnO}$ NPs has antioxidant effects on the alterations of testis induced by $\mathrm{BU}$ in the adult male mice as; ultrastructure study.

\section{Materials and Methods}

\section{Chemicals}

Myleran (busulfan) (1, 4-butanediol dimethanesulfonate), used as tablets (2 mg), was manufactured by Excella $\mathrm{GmbH}$ (Feucht, Germany). The BU dosage was prepared in normal saline after being dissolved in DMSO and then was administrated in a single (i.p) dose of $40 \mathrm{mg} / \mathrm{kg}$ (Mirhoseini et al., 2014).

Melatonin was purchased as $10 \mathrm{mg}$ tablets from Puritan's Pride (Holbrook, NY, USA). Mel dosage prepared in water and was administrated orally at doses of $20 \mathrm{mg} / \mathrm{kg} /$ day (Mirhoseini et al., 2014).

Zinc oxide nanoparticles were white powdered with particle size 10-30 nm (Catalog Number US 677450, SigmaAldrich, Saint Louis, MO, USA). The ZnO NP dosage was prepared in standered saline solution and administrated intraperitoneally at $5 \mathrm{mg} / \mathrm{kg} /$ day (Torabi et al., 2017).

\section{$\mathrm{ZnO}$ nanoparticle preparation and characterization}

$\mathrm{ZnO}$ nanoparticle powder was autoclaved and UV-sterilized overnight. The sterilized $\mathrm{ZnO}$ nanoparticles were suspended and ultra-sonicated (Bransonic ultrasonic cleaner 3210, 120W, Branson Ultrasonic Corporation, Danbury, CT, USA) for $15 \mathrm{~min}$ in pure ethanol (99.9\%, Samchun Chemicals, Seoul, Korea). The $\mathrm{ZnO}$ nanoparticles of Transmission electron microscopy images were observed in a Jeol JSM-6510 L.V SEM microscope using $30 \mathrm{KV}$ at EM Unit, Mansoura University, Egypt (Fig. 1).

\section{Experimental animals}

Thirty healthy male Swiss albino mice weighting 25-32 g were obtained from the Animal House of the Faculty of Pharmacy, Mansoura University, Egypt. They were housed in an artificially illuminated ( $12 \mathrm{~h}$ light $-12 \mathrm{~h}$ dark cycle), air-conditioned and temperature-controlled room (22$25^{\circ} \mathrm{C}$ ); the commercial laboratory chow and water were available for the animal's ad libitum. The experiment started after two weeks of acclimatization. All animals received humane care and compliance-with the guidelines of the Animal Care and Use Committee of Mansoura University. Code No. ZD180015.

\section{Experimental design}

After the acclimatization period, the animals were randomly assigned into six groups of five mice each.

Group 1 (control): mice received orally $0.9 \%$ saline solution for 75 days.

Group 2 (Mel): mice received oral doses of $20 \mathrm{mg} / \mathrm{kg} /$ day of Mel for 30 days.

Group 3 ( $\mathrm{ZnO} \mathrm{NPs})$ : mice injected daily i.p with $5 \mathrm{mg} / \mathrm{kg} /$ day of $\mathrm{ZnO}$ NPs for 30 days.

Group 4 (BU): mice injected i.p with a single dose of $\mathrm{Bu}$ $40 \mathrm{mg} / \mathrm{kg}$ and left for 45 days.

Group $5(\mathrm{BU}+\mathrm{Mel})$ : mice injected with a single dose of BU followed by Mel after 45 days of BU injection for 30 days.

Group 6 (BU+ ZnO NPs): mice injected with a single dose of $\mathrm{BU}$ followed by $\mathrm{ZnO} \mathrm{NPs}$ after 45 days of $\mathrm{BU}$ injection for 30 days.

\section{Investigated parameters}

\section{Light microscopic study}

In all experimental groups, the specimens of right side testis were removed and immediately washed in normal saline and fixed in $10 \%$ neutral buffered formalin. After fixation, the samples were dehydrated with an ascending ethanol series, cleared in xylene, embedded in paraffin wax and sectioned at $5 \mu \mathrm{m}$ thickness. Sections were stained with Masson's trichrome to demonstrate collagen fibers and examined under a bright- field Olympus microscope.

\section{Toluidine blue stain}

Semithin sections were cut with a diamond knife on an LKB microtome and mounted on slides, stained with toluidine blue and examined by a light microscope. 
Transmission electron microscopic investigations (TEM)

The ultrastructural investigations of the present work were mainly focused on the testis of male mice. Small pieces of the right testis were immediately separated and fixed in $4 \%$ glutaraldehyde in Dulbecco's modified phosphate buffered saline. Samples were washing and fixed in $1 \%$ osmium tetroxide for $1 \mathrm{~h}$ at room temperature. After that, they were dehydrated in an ethanol gradient, treated via propylene oxide, then embedded in Epon resin (Epon 812; Fluka Chemie, Switzerland) for TEM investigations. Ultrathin sections $(60-70 \mathrm{~nm})$ were cut with a diamond knife on an LKB microtome and mounted on slides, stained with uranyl acetate and lead citrate (Hall et al., 2012). The sections were then examined and photographed at $80 \mathrm{kV}$ using a JEOL 2100 Transmission Electron Microscope in the Electron Microscopic Unit at Faculty of Agriculture, Mansoura University, Mansoura, Egypt.

\section{Results}

Characterization of $\mathrm{ZnO}$ nanoparticles

Transmission electron microscopy (TEM) images showed that $\mathrm{ZnO}$ nanoparticle had a sub-100 $\mathrm{nm}$ size on average after the sterilisation process.

\section{Masson trichrome observations}

The effect of control, Mel or ZnO NPs on testis was further assessed by demonstrating the expression of Masson on the testis. Masson trichrome stained sections of control, Mel or $\mathrm{ZnO}$ NPs groups showed minor expression of collagen fibers at the boundary of seminiferous tubules and in between the tubules (Figs. 2A-2C). A section in the testis of the BU group showed intense positive expression of collagen fibers at the boundary of seminiferous tubules and in between the tubules. Busulfan (BU) was reported as cytotoxic drug associated with pulmonary toxicity, acute lung injury, chronic intestinal fibrosis that defined as the overgrowth, hardening and deposition of extracellular matrix components including collagen (Fig. 2D). Mel or ZnO NPs supplemented mice after $\mathrm{BU}$ treatment showed a mild expression of Masson trichrome staining (Figs. 2E and 2F).

\section{Toluidine blue observations}

Semithin sections in the testis of control, Mel and ZnO NPs groups stained with toluidine blue, showed seminiferous tubules lined by germinal epithelium, including several types of spermatogenic cells at different stages of spermatogenesis. Sertoli cells were resting on regular basement membranes ensheathed by flat myoid cells and nearly similar to the control. Normal spermatids and an increased number of sperms in the lumen of seminiferous tubule were also noticed (Figs. 3A-3C). The adjacent tubules showed wide intercellular spaces separating the germ cells in the BUtreated group. Degenerated Leydig cells in the interstitial spaces and congested blood vessel were observed. Few number and abnormal shape of spermatids were also seen. The lumen of seminiferous tubules of the BU treated group showed devoid of sperms (Fig. 3D). BU injected mice and supplemented with $\mathrm{Mel}$ or $\mathrm{ZnO}$ NPs groups improved the general histological picture of the testis. The architecture of seminiferous tubules showed nearly normal and observed the lining of germinal epithelium with well-organized spermatogenic layers at different stages of spermatogenesis. Sertoli cells were nearly normal in their structure. Moreover, seminiferous tubules with much sperms in their lumen and some Leydig cells in the interstitial spaces were also seen inbetween (Figs. 3E and 3F).

\section{Electron microscopic observations}

The ultrathin sections of control testis revealed that seminiferous tubules (S.T) were surrounded by thin basal lamina that contains flat myoid cells and lined by spermatogenic cells. The epithelium of spermatogenic cells showed the sequences of spermatogonia, spermatocytes, spermatids, Sertoli cells and germ cells in active spermatogenesis. Sertoli cells showed a distinct nucleus with prominent nucleoli and a cytoplasm containing numerous mitochondria, endoplasmic reticulum, lysosomes and lipid droplets (organelles) in an active state. It is located between spermatogenic cells and rested on the basement membrane of the seminiferous tubule. The spermatogonia rested on the basal lamina of the tubule, and had a rounded nucleus with prominent nucleoli and numerous mitochondria were seen in their cytoplasm (Fig. 4A). The primary spermatocytes were round in shape with central large rounded nuclei containing peripheral euchromatin condensation and heterochromatin; numerous mitochondria and lysosomes were also seen (Fig. 4B).

Round spermatid appeared with regular plasma membrane surrounded by acrosomal vesicle with normal

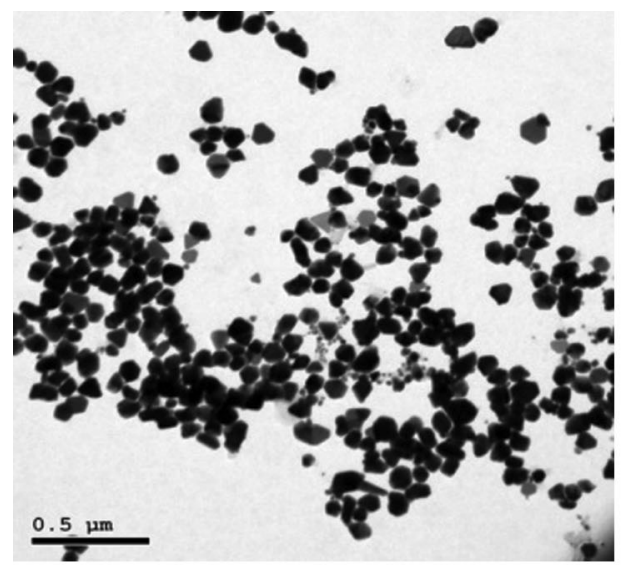

A

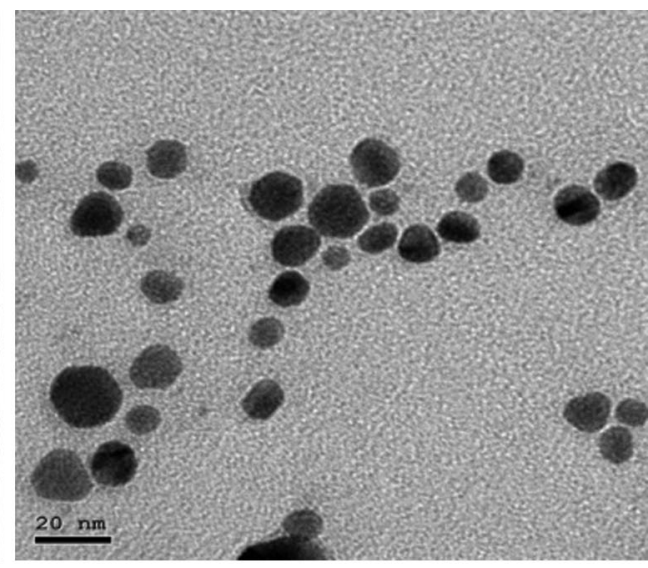

B
FIGURE 1. (A \& B): Characterization of the sterilized $\mathrm{ZnO}$ nanoparticle; $\mathrm{A}$ and B transmission electron microscopy (TEM) images of the $\mathrm{ZnO}$ nanoparticle. 


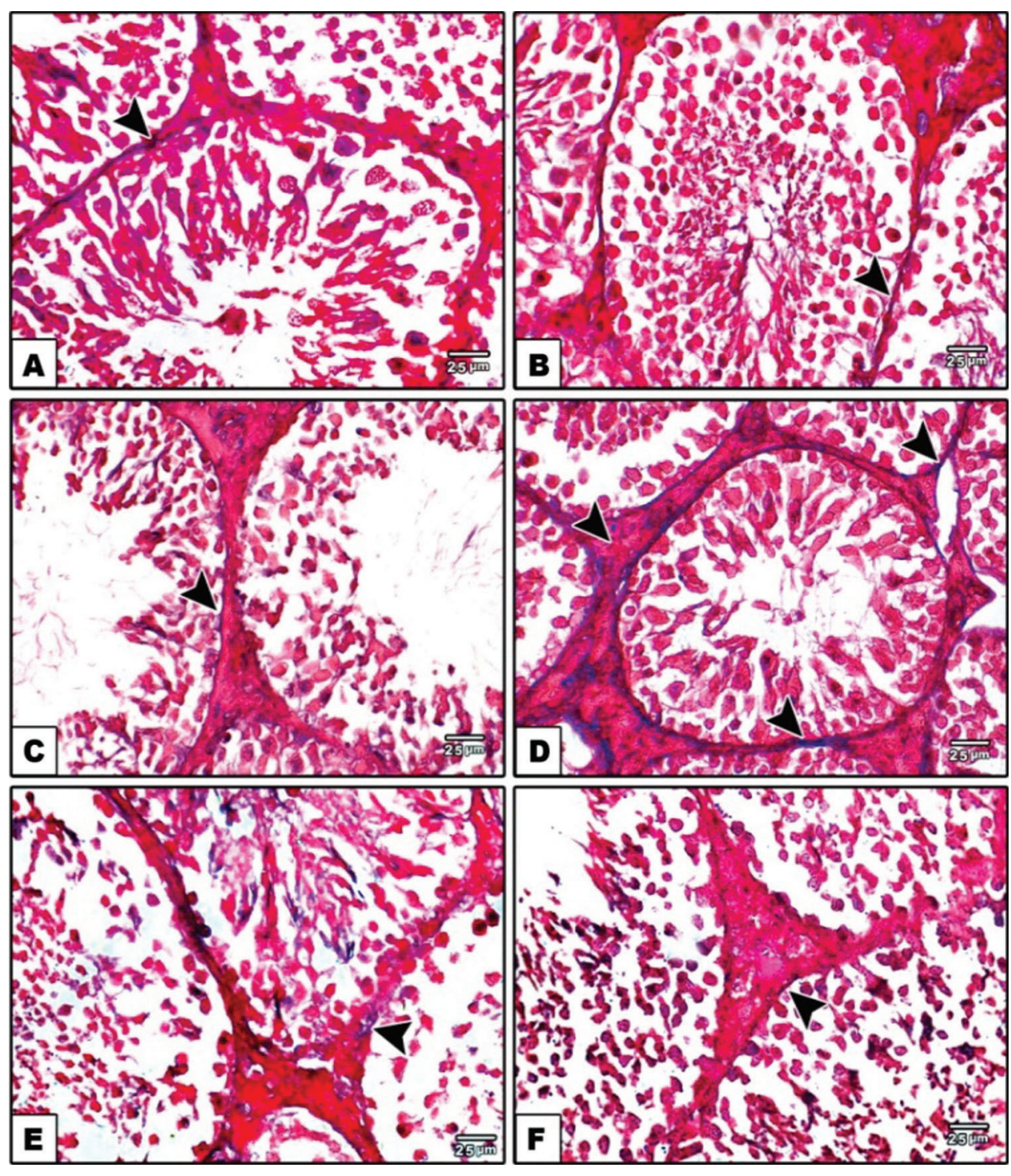

FIGURE 2. (A-F) Photomicrographs of mice testes in the control and different experimental groups. Masson trichrome staining. (A, B \& C) control, Mel and $\mathrm{ZnO}$ NPs showing minor collagen fibers (arrowhead); (D) $\mathrm{BU}$ group showing dense collagen fibers (arrowhead); (E) BU + Mel group showing minor collagen fiber (arrowhead); (F) BU + ZnO NPs group showing minor collagen fibers (arrowhead). round nucleus and nucleolus. Its cytoplasm contains welldeveloped mitochondria, ribosomes and Golgi apparatus (Fig. 4C). The tail of mature sperm in the middle piece showed that it is formed of central axoneme, dense outer fibers and mitochondrial sheath were around the dense fibers. The mitochondrial sheath was surrounded by the plasma membrane (Fig. 4D). The head of mature spermatozoa was elongated, containing an acrosomal cap covering the nucleus (Fig. 4E). The Leydig cell showed a regular plasma membrane with euchromatic nucleus and prominent nucleolus; its cytoplasm had many normal mitochondria and lysosomes (Fig. 4F).

On the other hand, transmission electron microscopic examination of the testicular tissues in BU-treated group revealed many alterations manifested by irregular thickened basal lamina and thick collagen fibers between myoid cells and basal lamina in focal area, the thickness of basal lamina causes low rate of sperm production. Some tubules showed reduced spermatogenesis (Figs. 5A-5C). Sertoli cells appeared with irregular plasma membrane and nucleus with disintegrated nucleoli and separated from basal lamina. The nucleus of Sertoli cell was irregular in shape and small in size (Fig. 5A). In this group, some seminiferous tubules showed impairment in the growth of spermatogenic cells, and the basal lamina surrounding the seminiferous tubules was irregular and tortuous. The presence of vacuoles was evident in the cytoplasm of Sertoli cells, spermatocytes and spermatids. In some germ cells, the membranes of the nuclei were swollen and vacuolated, including apoptosis in some germ cells. Spermatogonia and primary spermatocyte cells appeared abnormal architecture with degenerated nuclei in some cells and pyknotic in another (Figs. 5A and 5B). The cytoplasm of some germ cells contains clumped organelles with degenerated mitochondria and vacuoles (Fig. 5B). Round spermatids had distorted and degenerated nucleus, cytoplasm revealed deformed and vacuolated mitochondria (Fig. 5C). Some abnormalities of the acrosome of the elongated spermatids were seen, separation of the acrosome from the nucleus, a wide separation between the cells and many vacuoles were also observed (Fig. 5D). Also, a wide separation between the germ cells was observed (Fig. 5E). The BU-treated group showed damage of Leydig cell with degenerated nucleus, irregular plasma membrane. The cytoplasm showed degenerated mitochondria, many vacuoles and many lysosomes (Fig. 5F). 


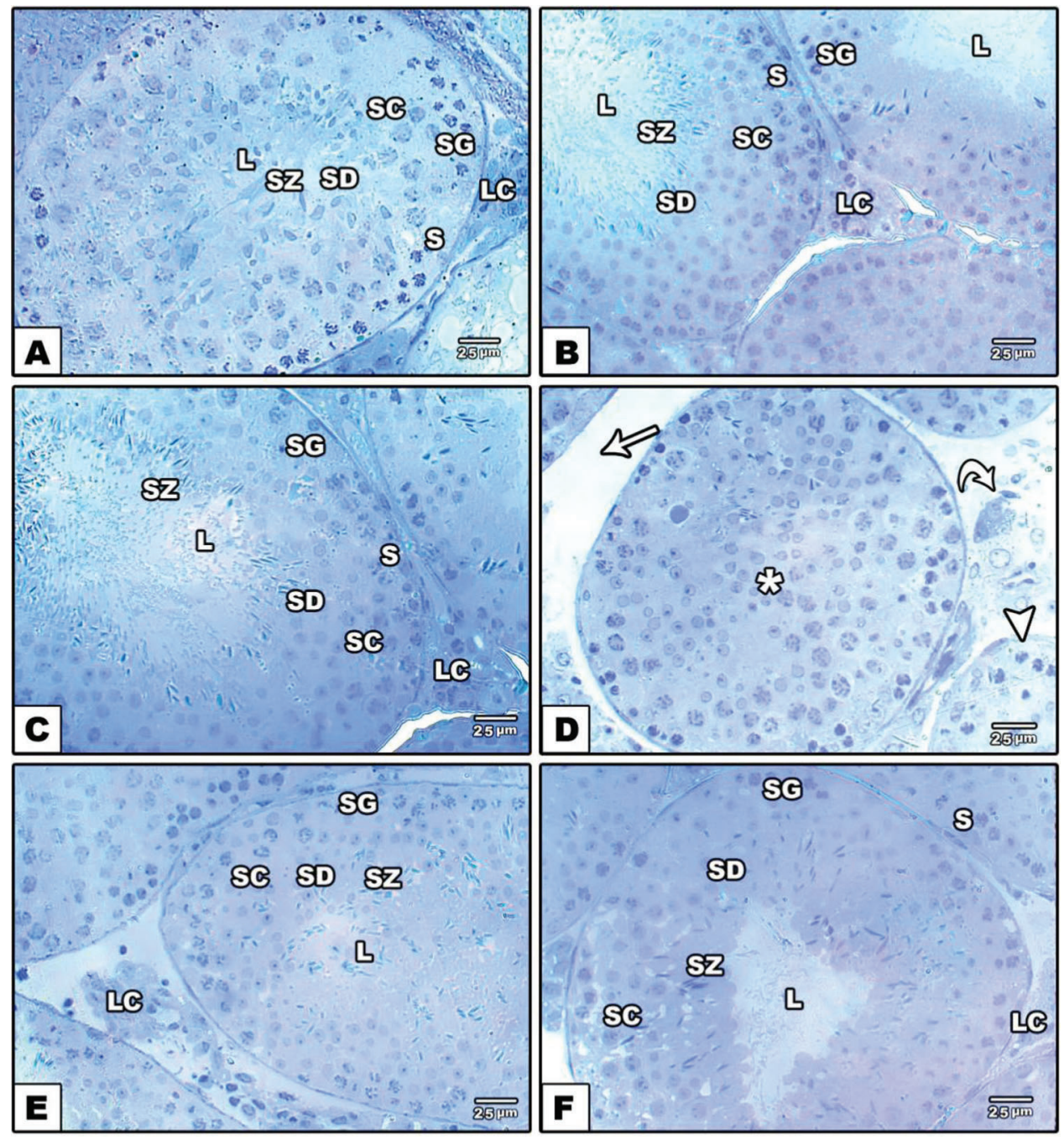

FIGURE 3. (A-F) Toluidine blue stained semithin sections in the testes of control and experimental groups. (A, B \& C) control, Mel and ZnO NPs showing well organized seminiferous tubules and arrangement of Sertoli cell resting on basement membrane spermatogonia, rest on intact basement membrane, large primary spermatocytes, round and elongated spermatids and numerous mature sperms are present in the lumen, normal Leydig cells.; (D) BU-treated group showing thin wall of seminiferous tubules, wide intertubular spaces with degenerated interstitial cells and reduced number of Leydig cells, no sperms found in lumen, destroyed most spermatogonia and spermatocytes cells; (E \& F) Mel or ZnO NPs supplemented after BU group showing recover seminiferous tubules surrounded by regular basement membrane, improve Leydig cells, spermatogonia, Sertoli cells, and increased volume of sperms in the lumen.

Abbreviations and symbols: Lumen (L), Leydig cells (LC), spermatogonia (SG), spermatozoa (SZ), separation of spermatogenic cell from the basement membrane (white arrowhead), Sertoli cells (S) and spermatocyte (SC), spermatid (SD), increased interstitial spaces (arrow), degenerated Leydig cells (curved arrows), no sperm in the lumen $\left(^{*}\right)$ and no spermatogenic process (arrowhead).

Electron micrographs of the group treated with BU and supplemented with Mel showed normal regular and thin basal lamina, revealing that Mel reduced the thickness of basal lamina in mice treated with BU. The standard architecture of Sertoli cells resting on the basal lamina with normal nucleus were also seen. All types of spermatogenic cells such as spermatogonia, primary spermatocytes and spermatids were normal structure and cytoplasm contains many mitochondria (Figs. 6A and 6B). Thin and regular basal lamina, normal spermatogonia with normal nucleus, primary spermatocytes and spermatids were normal structure also noticed (Fig. 6C). Round spermatids had normal oval nucleus, the nuclear membrane was thickened from one side forming acrosomal vesicles and minor cells has pyknotic nuclei. The lumen of seminiferous tubules showed increase number of normal sperms with dense stained nuclei covered by acrosome (Figs. 6D and 6E). Leydig cells had regular plasma membrane, nuclei with prominent nucleoli and the cytoplasm showed numerus mitochondria, rough endoplasmic reticulum and lysosomes (Fig. 6F).

Moreover, ultrathin sections of testicular tissues of mice treated with BU and supplemented with $\mathrm{ZnO}$ NPs showed marked improvement of spermatogonia, primary spermatocytes, Sertoli cells and spermatids. Thin and regular basal lamina and normal architecture of Sertoli cells resting on the basal lamina with normal nucleus were also seen (Figs. 7A and 7B). The round spermatids had normal nucleus and nucleolus with regular plasma membrane and covered with acrosomal vesicles, cytoplasm contains abundant mitochondria, ribosomes and lysosomes (Figs. 7C and 7E). The lumen of seminiferous tubules showed numerous of normal mature spermatozoa with normal acrosome and nucleus, cytoplasm contains many 

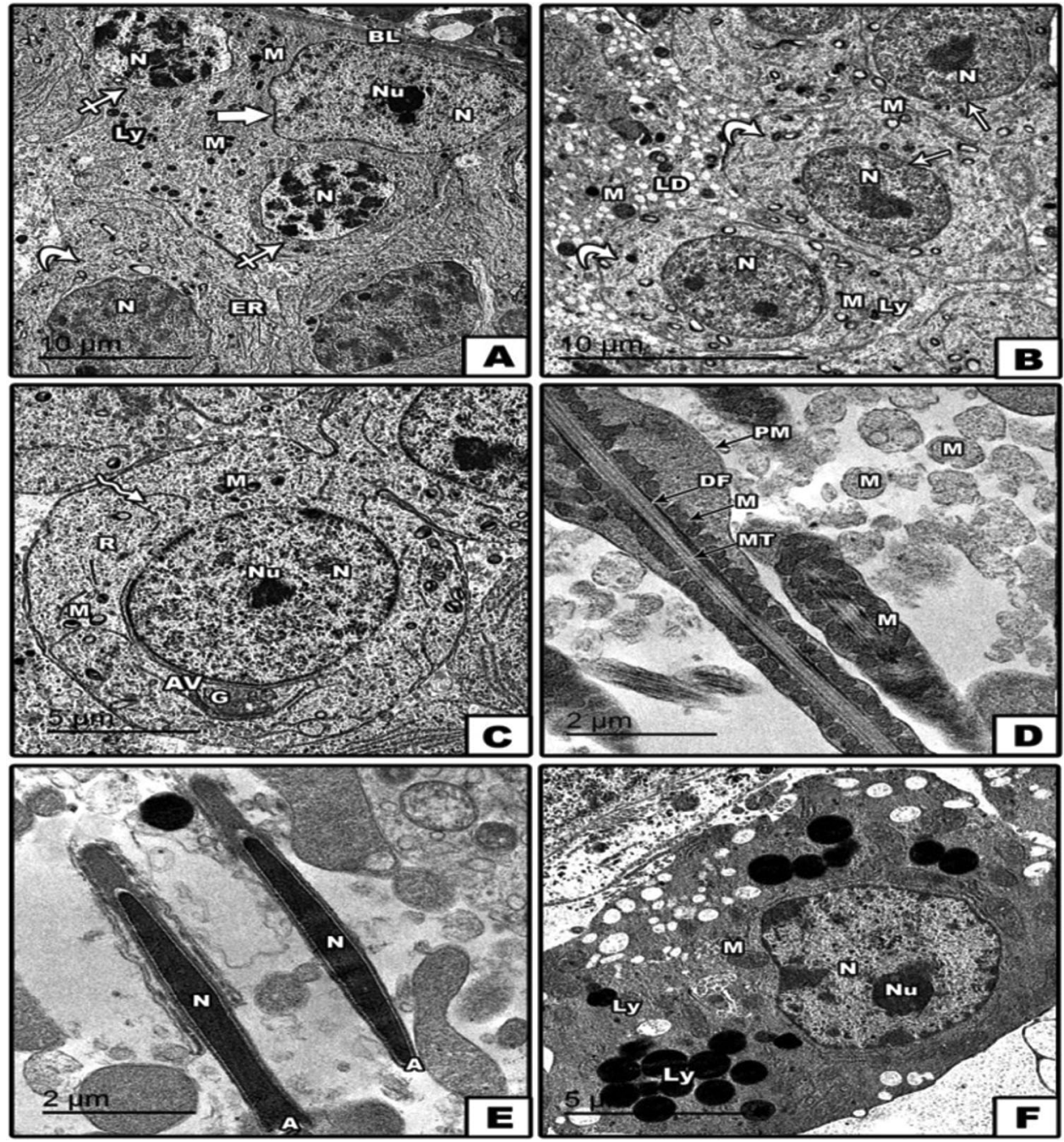

FIGURE 4. (A-F) Electron micrographs of seminiferous tubules of control mice: (A \& B) thin basal lamina (BL), normal appearance of Sertoli cell resting on basal lamina (Thick arrow), spermatogonia (closed arrow), Nucleus of Sertoli cell (N), nucleolus $(\mathrm{Nu})$, spermatocyte (curved arrow), lysosomes (Ly), mitochondria (M), endoplasmic reticulum (ER), lipid droplets (LD); (C) Spermatid (zigzag arrow), Nucleus of spermatid $(\mathrm{N})$, nucleolus $(\mathrm{Nu})$, Acrosomal vesicle (AV), mitochondria (M), Ribosome (R), Golgi apparatus (G); (D) Plasma membrane (PM), Dense fiber (DF), Microtubule (MT), mitochondria (M); (E) Acrosome (A), Nucleus of sperm (N); (F) Nucleus of Leydig cell (N) with nucleolus $(\mathrm{Nu})$, Lysosomes (Ly), mitochondria $(\mathrm{M})$. mitochondria and lysosome (Figs. 7D). Leydig cells had regular plasma membrane, nuclei with prominent nucleoli and the cytoplasm showed numerus mitochondria and lysosomes (Fig. 7F).

\section{Discussion}

Infertility is a disease of the reproductive system defined by the failure to achieve a clinical pregnancy after one year of regular unprotected sexual practice (Bashir et al., 2009). Male infertility risk factor such as age, smoking, obesity, exercise, diet, electronic devices, stress, scrotal temperature, reactive oxygen species and therapeutic drugs (Olayemi, 2010). Some therapeutic drugs such as alkylating agents (cyclophosphamide and busulfan) alter fertility, change organ structure, sex hormones, function and life quality (Tsuyoshi et al., 2015).

Busulfan (BU) is one of the alkylating agents widely used treating various cancers, leukemia, malignant lymphomas and autoimmune diseases (Gabrielsen and Tanrikut, 2016). Despite the many clinical applications of BU, it has critical complications as spermatogenesis disorder and infertility (Juenke et al., 2011).

Antioxidant agents neutralize free radicals and oxidative stress resulting from chemotherapeutic agents (Simone et al., 2007).
Melatonin (Mel) is a free radical scavenger that regulates various molecular pathways, such as inflammation, proliferation, apoptosis, and metastasis during different pathological conditions (Cui et al., 2017). Zinc possesses important properties, including its use in many applications and its flexibility in preparing it in different shapes and properties, making it a magic material (Padmavathy and Vijayaraghavan, 2008). It is an antioxidant and a vital trace element necessary to maintain germ cells. The high $\mathrm{Zn}$ concentrations in testis and accessory gonadal glands indicate a critical role in reproduction (Tuncer et al., 2011). ZnO NPs have potent antioxidants and can ameliorate reproductive function (Mohammadi et al., 2017; Torabi et al., 2017).

In the present results, Masson trichrome staining in BUtreated group showed dense collagen fibers at the boundary of seminiferous tubules and in between the tubules due to Busulfan (BU) was the first cytotoxic drug associated with pulmonary toxicity, acute lung injury, chronic intestinal fibrosis that characterized by overgrowth, hardening and is attributed to excess deposition of extracellular matrix components including collagen (Oliner et al., 1961; TA Wynn, 2008). These results were in agreement with Hussein et al. (2015). It could be explained by the released factors from the macrophages and $\mathrm{T}$ lymphocytes, stimulating collagen deposition. Moreover, activated macrophages could 

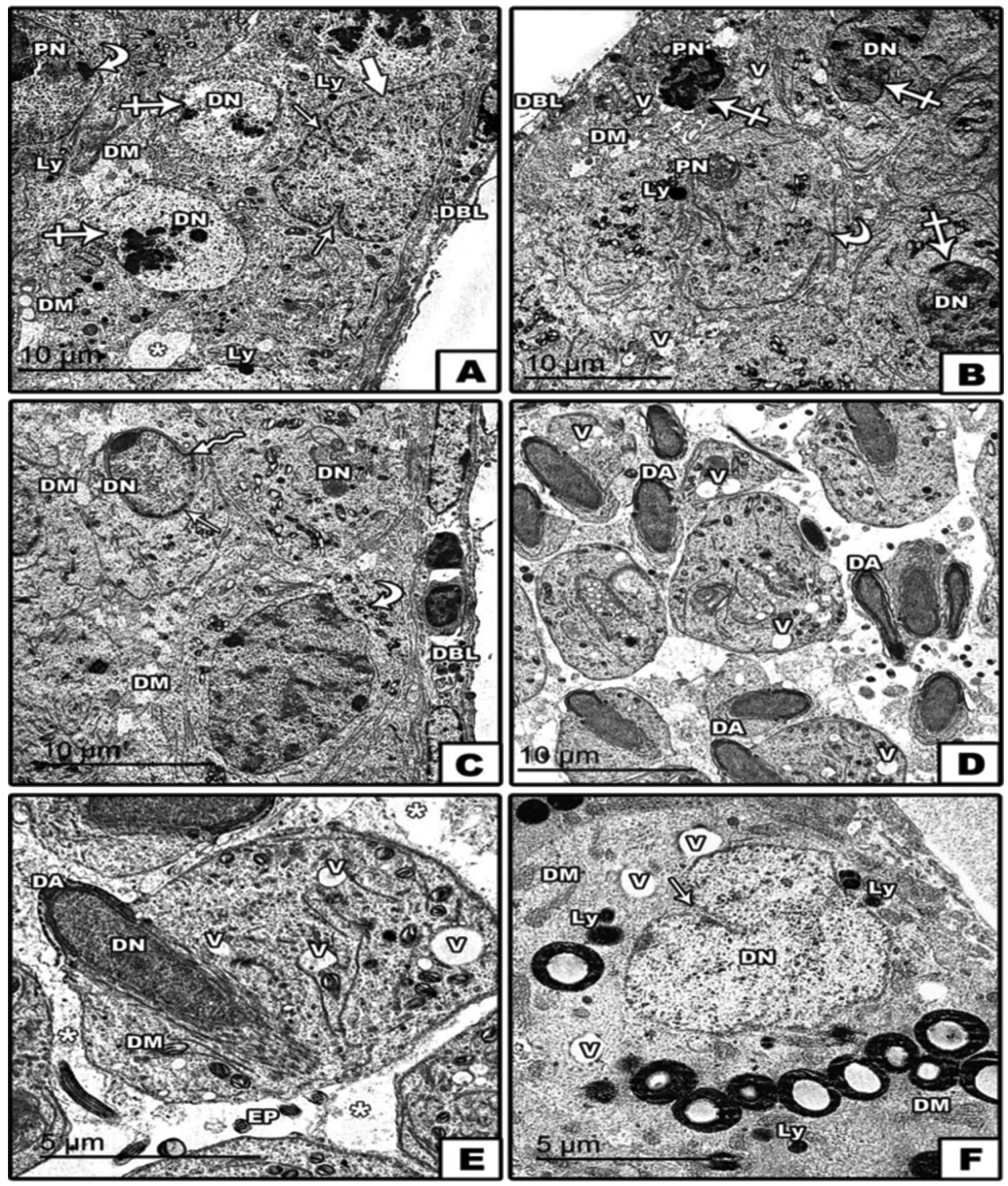

FIGURE 5. (A-F): Electron micrographs of seminiferous tubules of BU-treated mice: (A \& B) Degenerated basal lamina (DBL) (thickened and irregular basal lamina of the seminiferous tubule), many vacuoles (V), irregular Sertoli cell (thick arrow), spermatogonia (closed arrow) with degenerated nucleus (DN), Nucleus of Sertoli cell (N), nucleolus $(\mathrm{Nu})$, spermatocyte (curved arrow) with Pyknotic nucleus (PN), lysosome (Ly), Degenerated mitochondria (DM), wide separation between cells (asterisk); (C) Spermatid (zigzag arrow) with degenerated nucleus $(\mathrm{N})$ and irregular plasma membrane (thin arrow), degenerated mitochondria (DM); (D) malformation of sperms with degenerated acrosome (DA), many vacuoles $(\mathrm{V})$; (E) sperm with degenerated acrosome (DA) and with degenerated nucleus (DN), Degenerated mitochondria (DM), wide separation between cells (asterisk), vacuoles (v), end piece (EP); (F) Ledying cell with degenerated nucleus (DN) and irregular plasma membrane (thin arrow) ,degenerated mitochondria (DM), vacuoles (V), lysosome (ly). stimulate the epidermal growth factor (EGF) which induces granulation tissue formation and fibrosis (Murray and Wynn, 2011). In the present results, Mel. supplemented group after $\mathrm{BU}$, resulted in minor collagen fibers. This consistent with Ilbey et al. (2009); Mel improved histopathological changes and this due to its antioxidant and anti-apoptotic properties (Bejarano et al., 2009; Mirhoseini et al., 2014). Also, the present results showed few of collagen fibers in $\mathrm{ZnO}$ NPs supplemented group after BU. These go parallel with Meligy et al., 2019; and this protection due to its antioxidant and anti-apoptotic properties of Zn (Afifi et al., 2015; Maremanda et al., 2016).

It is well known that normal Sertoli and Leydig cell functions provide a principle activation and maintenance of spermatogenesis. In the current work, the ultrastructure study of testicular tissue elucidated that BU altered seminiferous tubules and changed the normal structure of Sertoli cells. These results were consistent with Anan et al. (2018) who observed the impairment of Sertoli cells because of the increased oxidative stress in the testes induced by chemotherapeutic agent. Also, the present results revealed that basal lamina surrounded seminiferous tubules plays an important role in spermatogenesis, which was found thickened and irregular and this thickness would cause a decreased rate of sperm production. Our results were consistent with who stated that stimulation of myoid cells that produce more collagen and extracellular matrix leads to thicken and irregular, basal lamina (Sakr et al., 2012). The previous authors reported that the change succeeded thickened basal lamina in its binding properties, impairing spermatogenesis by limiting access of different hormones and paracrine factors regulating sperm production (Aitken and Roman, 2009). These alterations due to BU exposure caused by over production of ROS. Moreover, degeneration of Leydig cells may impair the function of secreting hormone in BU group; this was in agreement with who revealed that chemotherapeutic drugs increased free radicals, damaging the Leydig cells (Colpi et al., 2004; Hutson, 2006).

On the other hand, Mel has protective role against hazardous effects of BU. In Mel supplemented group after BU showed normal spermatogenic cells, Sertoli cells and thin basal lamina. These results parallel those who reported that Mel restores almost normal basal lamina by decreasing the thickness and provided protection for spermatogenesis 

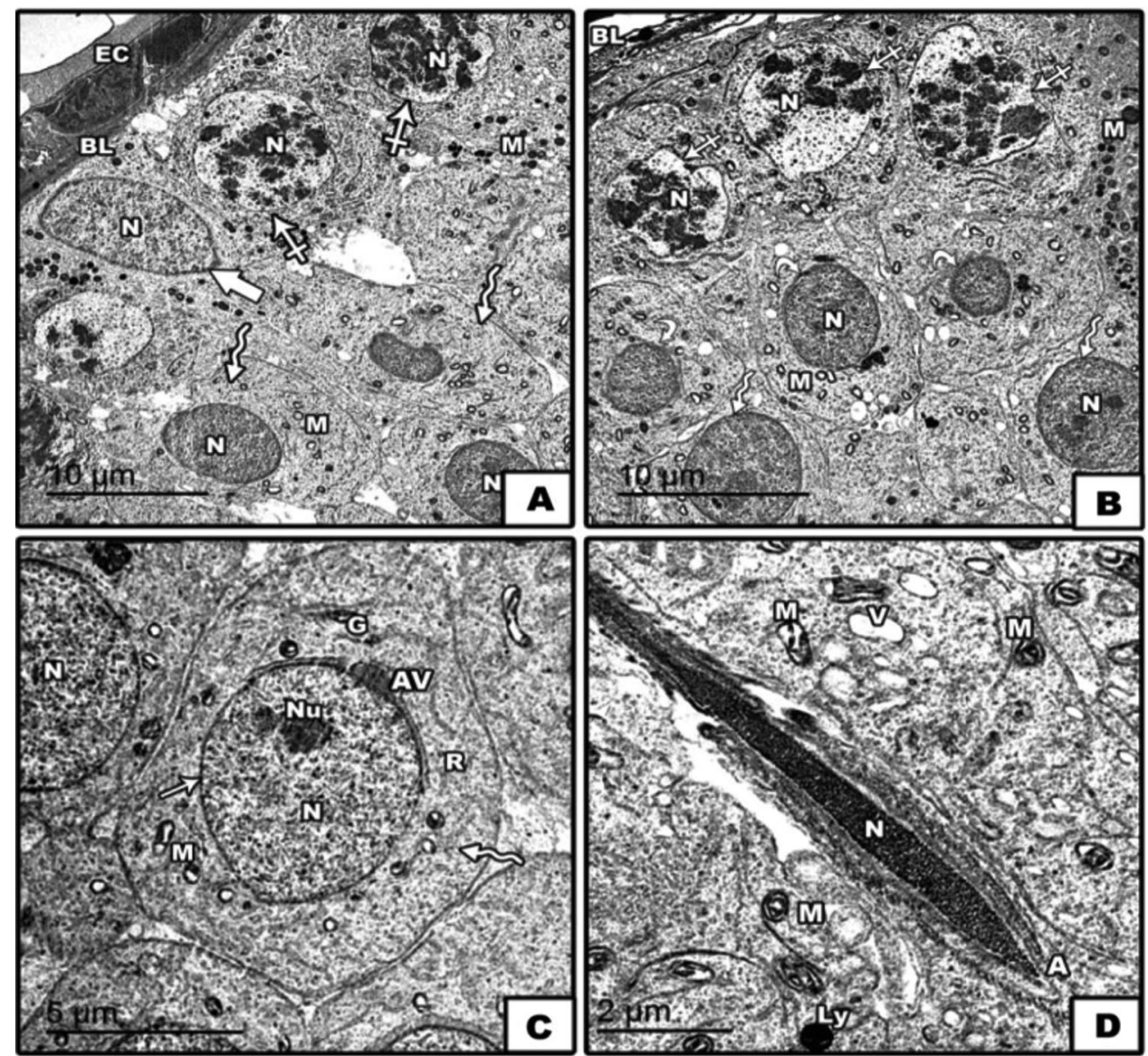

FIGURE 6. (A-F): Electron micrographs of seminiferous tubules of BU-treated mice supplemented with Mel; (A, B \& C) thin basal lamina (BL), normal appearance of Sertoli cell
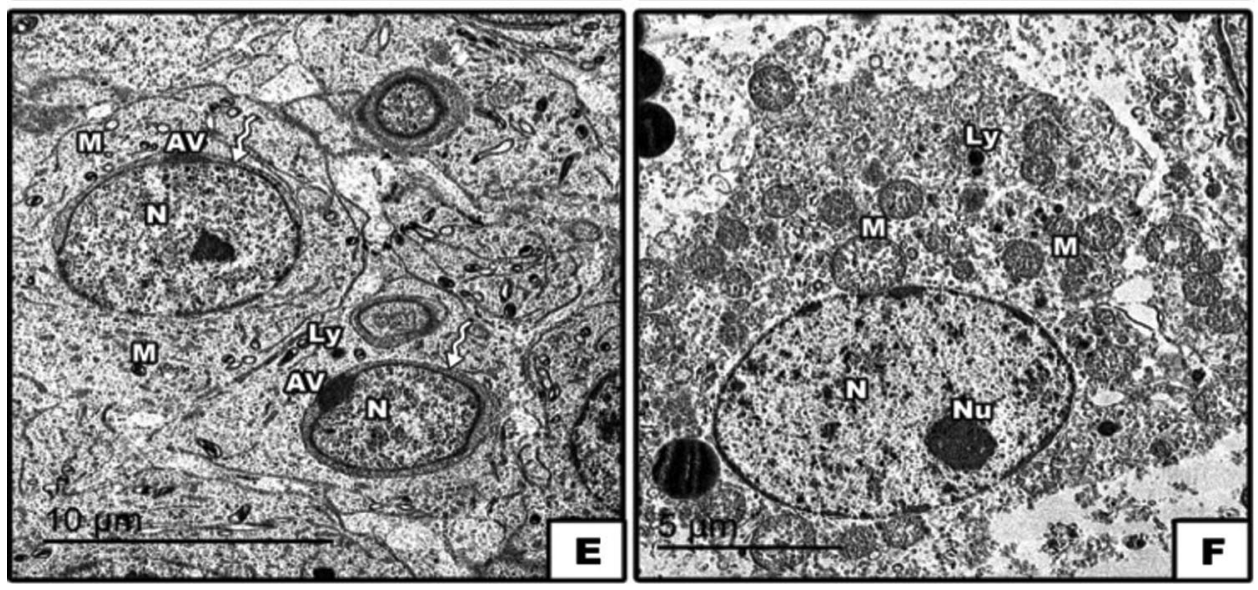
resting on basal lamina (Thick arrow), normal spermatogonia (closed arrow), Nucleus of Sertoli cell (N), nucleolus $(\mathrm{Nu})$, normal spermatocyte (curved arrow), mitochondria (M); normal spermatid (zigzag arrow); (D) many sperm with nucleus $(\mathrm{N})$ and acrosome (A), spermatid (zigzag arrow); (E) many elongated sperm with nucleus $(\mathrm{N})$ and acrosome (A); many mitochondria (M); (F) nucleus of normal Ledying cell $(\mathrm{N})$, lysosome (Ly), mitochondria (M), rough endoplasmic reticulum (RER).

(Zhang et al., 2012). In another study, Espino et al. (2010) was demonstrated that Mel also prevents oxidative stress and apoptosis of mitochondrial pathways. Antioxidant could protect living cells from BU damage by improving the deleterious effect of free radicals, so Mel has an ability of scavenging free radicals and regulate gene transcription for some antioxidant enzymes (Bharti et al., 2011). Also, the present results investigated the protective effects of $\mathrm{ZnO}$ NPs against hazardous effects of BU showed marked improvement of spermatogonia, spermatocytes, Sertoli cells, leydig cells, thin and regular basal lamina. These results agreed with those who reported that $\mathrm{ZnO}$ NPs improved the adverse effects of chemotherapeutic agent (Anan et al., 2018). It was showed that $\mathrm{ZnO}$ NPs have anti-apoptotic activates and Zn supplementation gives a protective effect against molecules apoptosis (Tian et al., 2009). Authors stated that one of potential mechanisms of decreased apoptosis was suppressing caspase enzyme activity. The other explanation of such findings was that $\mathrm{ZnO}$ NPs increased mitochondrial functions resulting in decreased apoptosis- inducing factor and cytochrome $\mathrm{c}$ then preventing apoptosis (Kaufmann and Hengartner, 2001). Also, in 2017, Torabi et al. (2017) reported that $\mathrm{ZnO}$ NPs have powerful antioxidant properties and can improve the reproductive function. 

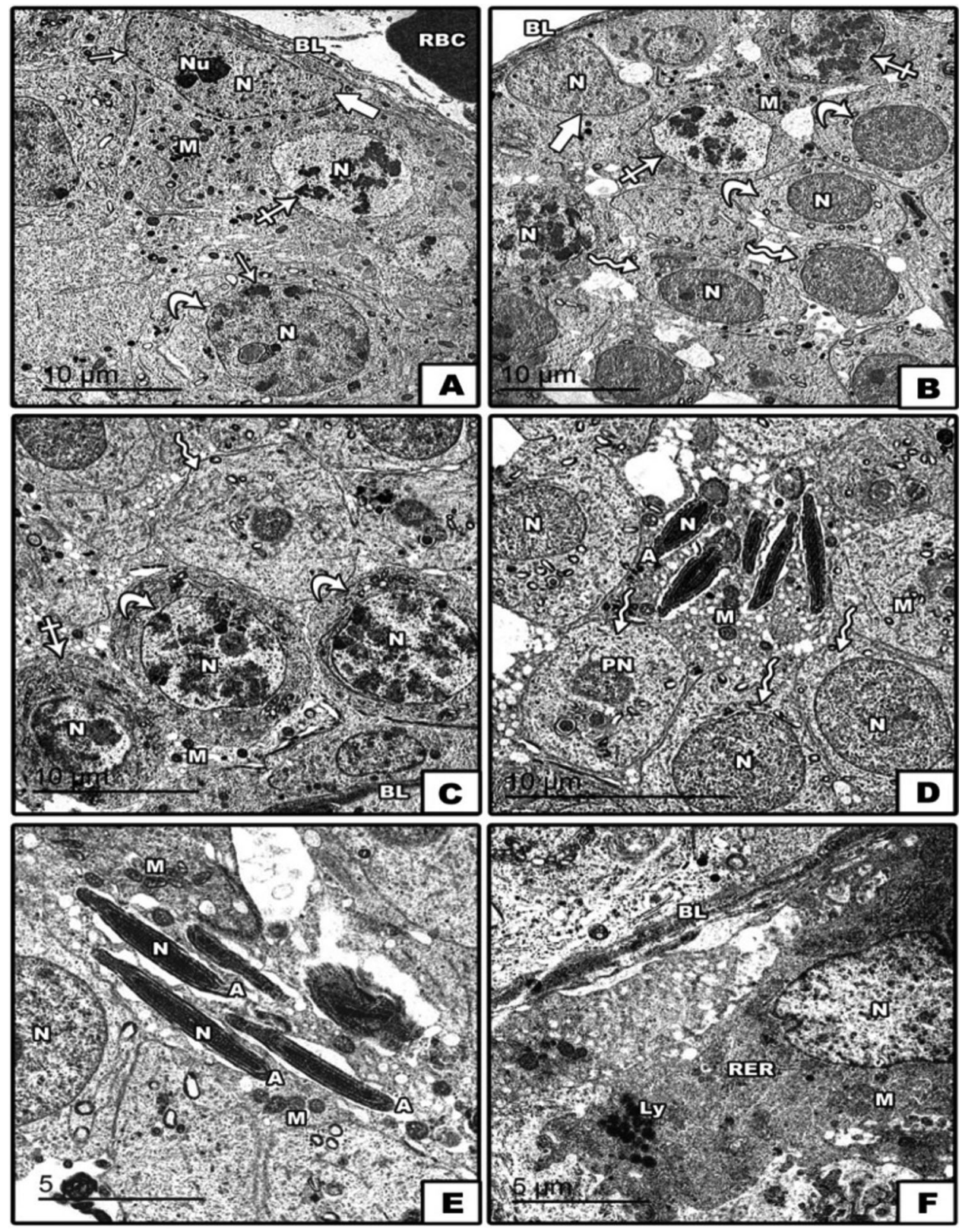

FIGURE 7. (A-F): Electron micrographs of mice seminiferous tubules of BU-treated mice supplemented with $\mathrm{ZnO}$ NPs: (A, B) thin basal lamina (BL), normal appearance of Sertoli cell resting on basal lamina (Thick arrow), normal spermatogonia (closed arrow), Nucleus of Sertoli cell $(\mathrm{N})$, nucleolus $(\mathrm{Nu})$, normal spermatocyte (curved arrow), mitochondria $(\mathrm{M})$; normal spermatid (zigzag arrow); (C, E) Spermatid (zigzag arrow), Nucleus of spermatid $(\mathrm{N})$, nucleolus $(\mathrm{Nu})$, Acrosomal vesicle (AV), mitochondria (M), Ribosome (R), Golggi apparatus (G); (D) mature sperm with nucleus $(\mathrm{N})$ and acrosome (A), lysosomes (Ly), mitochondria (M); (F) nucleus of normal Ledying cell $(\mathrm{N})$ with normal nucleolus $(\mathrm{Nu})$, lysosome (Ly), mitochondria (M).

\section{Conclusion}

In conclusion, chemotherapeutic drugs exert harmful effects on the reproductive system, degenerative changes of the testis of albino mice and arrested spermatogenic activity through several pathways. BU induced histological and ultrastructural damage of the testis. Moreover, the present results could be concluded that $\mathrm{Mel}$ or $\mathrm{ZnO}$ NPs can protect the testicular tissue against ultrastructural alterations induced by $\mathrm{BU}$ by its antioxidant and anti-apoptotic effects.

Acknowledgement: The authors would like to thank the Deanship of Scientific Research at Taif University for funding this work through Taif University Researchers Supporting Project No. (TURSP-2020/222), Taif University, Taif, Saudi Arabia.

Author Contributions: E.S.F., S.A.M. and A.M.A. suggested the work protocol, interpreted the results and revised the manuscript; E.S.F., A.M.A. performed the laboratory experiments, A.M.A., and E.S.F. prepared the manuscript; F.A., and E.F. for funding acquisition, resources and data analysis. All authors have read and agreed to the published version of the manuscript.

Data Availability Statement: The data generated or investigated during this study are obtainable from the corresponding author on reasonable request.

Ethics Approval: All experimental procedures were approved and supervised by the Institutional Ethical Committee for the care and use of laboratory animals in the Faculty of Science, Mansoura University, Egypt. All efforts were made to minimize animal suffering.

Funding Statement: This study was funded by Taif University Researchers Supporting Project No. TURSP-2020/222, Taif University, Taif, Saudi Arabia.

Conflicts of Interest: The authors declare that they have no conflicts of interest to report regarding the present study. 


\section{References}

Afifi M, Almaghrabi OA, Kadasa NM (2015). Ameliorative effect of zinc oxide nanoparticles on antioxidants and sperm characteristics in streptozotocin-induced diabetic rat testes. BioMed Research International 2015: 1-6.

Aitken RJ, Roman SD (2009). Antioxidant systems and oxidative stress in the testes. Molecular Mechanisms in Spermatogenesis 636: 154-171.

Anan HH, Zidan RA, El-Baset SAA, Ali MM (2018). Ameliorative effect of zinc oxide nanoparticles on cyclophosphamide induced testicular injury in adult rat. Tissue and Cell 54: 80-93.

Bashir A, Tahir M, Samee W, Munir B (2009). Effects of Tribulus terrestris on testicular development of immature albino rats. Biomedica 25: 63-68.

Bejarano I, Redondo PC, Espino J, Rosado JA, Paredes SD et al. (2009). Melatonin induces mitochondrial-mediated apoptosis in human myeloid HL-60 cells. Journal of Pineal Research 46: $392-400$.

Bharti VK, Srivastava R, Subramaian P, Spence DW, Pandi-Perumal $S$ et al. (2011). Cerebral epiphyseal proteins and melatonin modulate the hepatic and renal antioxidant defense of rats. International Journal of Nephrology 2011: 1-5.

Brinster CJ, Ryu BY, Avarbock MR, Karagenc L, Brinster RL et al. (2003). Restoration of fertility by germ cell transplantation requires effective recipient preparation. Biology of Reproduction 69: 412-420.

Chen X, Hao A, Li X, Du Z, Li H et al. (2016). Melatonin inhibits tumorigenicity of glioblastoma stem-like cells via the AKTEZH2-STAT3 signaling axis. Journal of Pineal Research 61: 208-217.

Colpi G, Contalbi G, Nerva F, Sagone P, Piediferro G (2004). Testicular function following chemo-radiotherapy. European Journal of Obstetrics \& Gynecology and Reproductive Biology 113: S2-S6.

Cui Y, Ren L, Li B, Fang J, Zhai Y et al. (2017). Melatonin Relieves busulfan-induced spermatogonial stem cell apoptosis of mouse testis by inhibiting endoplasmic reticulum stress. Cellular Physiology and Biochemistry 44: 2407-2421.

Delessard M, Saulnier J, Rives A, Dumont L, Rondanino C, Rives N (2020). Exposure to chemotherapy during childhood or adulthood and consequences on spermatogenesis and male fertility. International Journal of Molecular Sciences 21: 1454.

Elgazar V, Razanov V, Stoltenberg M, Hershfinkel M, Huleihel M et al. (2005). Zinc-regulating proteins, ZnT-1, and metallothionein I/II are present in different cell populations in the mouse testis. Journal of Histochemistry \& Cytochemistry 53: 905-912.

Espino J, Bejarano I, Redondo PC, Rosado JA, Barriga C et al. (2010). Melatonin reduces apoptosis induced by calcium signaling in human leukocytes: Evidence for the involvement of mitochondria and Bax activation. Journal of Membrane Biology 233: 105-118.

Fallah A, Mohammad-Hasani A, Colagar AH (2018). Zinc is an essential element for male fertility: A review of $\mathrm{Zn}$ roles in men's health, germination, sperm quality, and fertilization. Journal of Reproduction \& Infertility 19: 69.

Gabrielsen J, Tanrikut C (2016). Chronic exposures and male fertility: The impacts of environment, diet, and drug use on spermatogenesis. Andrology 4: 648-661.

Gurunathan S, Kang MH, Kim JH (2020). Role and therapeutic potential of melatonin in the central nervous system and cancers. Cancers 12: 1567.
Haghi-Aminjan H, Asghari MH, Farhood B, Rahimifard M, Hashemi Goradel N et al. (2018). The role of melatonin on chemotherapy-induced reproductive toxicity. Journal of Pharmacy and Pharmacology 70: 291-306.

Hall DH, Hartwieg E, Nguyen KC (2012). Modern electron microscopy methods for C. elegans. Methods in Cell Biology 107: 93-149.

Hussein YM, Mohamed RH, Shalaby SM, Abd El-Haleem MR, Abd El Motteleb DM (2015). Anti-oxidative and anti-apoptotic roles of spermatogonial stem cells in reversing cisplatininduced testicular toxicity. Cytotherapy 17: 1646-1654.

Hutson JC (2006). Physiologic interactions between macrophages and Leydig cells. Experimental Biology and Medicine 231: $1-7$.

Ilbey YO, Ozbek E, Simsek A, Otunctemur A, Cekmen M, Somay A (2009). Potential chemoprotective effect of melatonin in cyclophosphamide-and cisplatin-induced testicular damage in rats. Fertility and Sterility 92: 1124-1132.

Inhorn MC, Patrizio P (2015). Infertility around the globe: new thinking on gender, reproductive technologies and global movements in the 21 st century. Human Reproduction Update 21: 411-426.

Juenke JM, Miller KA, Mcmillin GA, Johnson-Davis KL (2011). An automated method for supporting busulfan therapeutic drug monitoring. Therapeutic Drug Monitoring 33: 315-320.

Kaufmann SH, Hengartner MO (2001). Programmed cell death: Alive and well in the new millennium. Trends in Cell Biology 11: 526-534.

Keerthana S, Kumar A (2020). Potential risks and benefits of zinc oxide nanoparticles: A systematic review. Critical Reviews in Toxicology 50: 47-71.

Kim JH, Jeong SJ, Kim B, Yun SM, Choi DY et al. (2012). Melatonin synergistically enhances cisplatin-induced apoptosis via the dephosphorylation of ERK/p90 ribosomal S6 kinase/heat shock protein 27 in SK-OV-3 cells. Journal of Pineal Research 52: 244-252.

Kong PJ, Byun JS, Lim SY, Lee JJ, Hong SJ et al. (2008). Melatonin induces Akt phosphorylation through melatonin receptorand PI3K-dependent pathways in primary astrocytes. Korean Journal of Physiology \& Pharmacology 12: 37-41.

Li B, He X, Zhuang M, Niu B, Wu C et al. (2018). Melatonin ameliorates busulfan-induced spermatogonial stem cell oxidative apoptosis in mouse testes. Antioxidants \& Redox Signaling 2018: 385-400.

Madhumitha G, Elango G, Roopan SM (2016). Biotechnological aspects of $\mathrm{ZnO}$ nanoparticles: Overview on synthesis and its applications. Applied Microbiology and Biotechnology 100: $571-581$.

Mao L, Yuan L, Slakey LM, Jones FE, Burow ME, Hill SM (2010). Inhibition of breast cancer cell invasion by melatonin is mediated through regulation of the p38 mitogen-activated protein kinase signaling pathway. Breast Cancer Research 12: R107.

Maremanda KP, Khan S, Jena G (2016). Role of zinc supplementation in testicular and epididymal damages in diabetic rat: involvement of Nrf2, SOD1, and GPX5. Biological Trace Element Research 173: 452-464.

Maroufi NF, Vahedian V, Hemati S, Rashidi M, Akbarzadeh M et al. (2020). Targeting cancer stem cells by melatonin: Effective therapy for cancer treatment. Pathology-Research and Practice 216: 152919.

Meligy FY, Abo Elgheed AT, Alghareeb SM (2019). Therapeutic effect of adipose-derived mesenchymal stem cells on 
Cisplatin induced testicular damage in adult male albino rat. Ultrastructural Pathology 43: 28-55.

Menéndez-Menéndez J, Martínez-Campa C (2018). Melatonin: An anti-tumor agent in hormonedependent cancers. International Journal of Endocrinology 2018.

Mirhoseini M, Saki G, Hemadi M, Khodadadi A, Asl JM (2014). Melatonin and testicular damage in busulfan treated mice. Iranian Red Crescent Medical Journal 16: 1-6.

Mohammadi T, Hoveizi E, Khajehpour L, Jelodar Z (2017). Protective effects of zinc oxide nanoparticles on testis histological structure in cyclophosphamide treated adult mice. Journal of Mazandaran University of Medical Sciences 26: 19-27.

Murray PJ, Wynn TA (2011). Protective and pathogenic functions of macrophage subsets. Nature Reviews Immunology 11: 723-737.

Naja M, Salehi E, Farhood B, Nashtaei MS, Hashemi Goradel N et al. (2019). Adjuvant chemotherapy with melatonin for targeting human cancers: A review. Journal of Cellular Physiology 234: 2356-2372.

Olayemi F (2010). Review on some causes of male infertility. African Journal of Biotechnology 9: 1-8.

Oliner H, Schwartz R, Rubio F, Dameshek W (1961). Interstitial pulmonary fibrosis following busulfan therapy. The American Journal of Medicine 131: 134-139.

Padmavathy N, Vijayaraghavan R (2008). Enhanced bioactivity of $\mathrm{ZnO}$ nanoparticles-An antimicrobial study. Science and Technology of Advanced Materials 9: 035004.

Poganitsch-Korhonen M, Masliukaite I, Nurmio M, Lähteenmäki P, Van Wely $M$ et al. (2017). Decreased spermatogonial quantity in prepubertal boys with leukaemia treated with alkylating agents. Leukemia 31: 1460-1463.

Reiter RJ, Tan DX, Herman TS, Thomas Jr CR (2004). Melatonin as a radioprotective agent: A review. International Journal of Radiation Oncology ${ }^{\star}$ Biology ${ }^{\star}$ Physics 59: 639-653.

Sakr SA, Mahran HA, Abo-El-Yazid SM (2012). Effect of fenugreek seeds extract on cyclophosphamide-induced histomorphometrical, ultrastructural and biochemical changes in testes of albino mice. Toxicology and Industrial Health 28: 276-288.

Simone Ii CB, Simone NL, Simone V, Simone CB (2007). Antioxidants and other nutrients do not interfere with chemotherapy or radiation therapy and can increase kill and increase survival, Part 2. Alternative Therapies in Health and Medicine 13: 22-29.
Suresh J, Pradheesh G, Alexramani V, Sundrarajan M, Hong SI (2018). Green synthesis and characterization of zinc oxide nanoparticle using insulin plant (Costus pictus D. Don) and investigation of its antimicrobial as well as anticancer activities. Advances in Natural Sciences: Nanoscience and Nanotechnology 9: 015008.

Tan DX, Manchester LC, Terron MP, Flores LJ (2007). One molecule, many derivatives: A never-ending interaction of melatonin with reactive oxygen and nitrogen species? Reiter Journal of Pineal Research 42: 28-42.

Tian L, Zhu F, Ren H, Jiang J, Li W (2009). Effects of nano-zinc oxide on antioxidant function in broilers. Chinese Journal of Animal Nutrition 21: 534-539.

Torabi F, Shafaroudi MM, Rezaei N (2017). Combined protective effect of zinc oxide nanoparticles and melatonin on cyclophosphamide-induced toxicity in testicular histology and sperm parameters in adult Wistar rats. International Journal of Reproductive BioMedicine 15: 403.

Tsuyoshi H, Orisaka M, Fukuda S, Hattori K, Tsang BK et al. (2015). Protective effect of dienogest on chemotherapy-induced reduced fertility in female rats. Steroids 93: 1-7.

Tuncer I, Sunar F, Toy H, Baltaci A, Mogulkoc R (2011). Histological effects of zinc and melatonin on rat testes. Bratislavske Lekarske Listy 112: 425-427.

Umar H, Kavaz D, Rizaner N (2019). Biosynthesis of zinc oxide nanoparticles using Albizia lebbeck stem bark, and evaluation of its antimicrobial, antioxidant, and cytotoxic activities on human breast cancer cell lines. International Journal of Nanomedicine 14: 87.

Wang J, Hao H, Yao L, Zhang X, Zhao S et al. (2012). Melatonin suppresses migration and invasion via inhibition of oxidative stress pathway in glioma cells. Journal of Pineal Research 53: 180-187.

Witt-Enderby PA, Radio NM, Doctor JS, Davis VL (2006). Therapeutic treatments potentially mediated by melatonin receptors: Potential clinical uses in the prevention of osteoporosis, cancer and as an Page 12/20 adjuvant therapy. Journal of Pineal Research 41: 297-305.

Wynn TA (2008). Cellular and molecular mechanisms of fibrosis. Journal of Pathology 214: 199-210.

Zhang K, Lv Z, Jia X, Huang D (2012). Melatonin prevents testicular damage in hyperlipidaemic mice. Andrologia 44: 230-236. 